\title{
CLINICAL QUALITY CONTROL OF MAMMOGRAMS EVALUATED IN A BRAZILIAN TERTIARY HOSPITAL
}

Leonardo R. Soares¹, Rosemar M. S. Rahal', Victória C. J. Queiroz¹, Erika C. Aquino', Rosangela S. Correa1', Danielle C. N. Rodrigues ${ }^{1}$, Ruffo Freitas-Junior ${ }^{1}$

${ }^{1}$ CORA - Centro Avançado de Diagnóstico da Mama, Universidade Federal de Goiás - Goiânia (GO), Brazil.

Objective: To evaluate the clinical quality of mammograms performed in users of the Unified Health System (SUS), referred to a tertiary hospital. Methods: A prospective study whose unit of observation was the mammograms of women referred for consultation in a Breast Cancer Resource Center, located in the city of Goiânia, Brazil, from May to October 2017. Mammograms for screening or for diagnosis were included, performed within six months prior to study inclusion. The clinical quality of the mammograms was evaluated using 40 variables per exam, related to the identification, the technique of the exam, the executing equipment, the radiological findings, the exam report and the mammary positioning. For these last variables, a comparison was made according to the origin of the exam (public vs. private network). Results: A total of 4560 items of clinical image quality were evaluated in 114 women whose mean age was 50.6 years. Of the total items analysed, there were 660 failures (14.47\%), and $443(67.12 \%)$ failures were related to breast positioning. Among the positioning failures, the absence of visualization of the pectoralis major muscle (86.8\%) and the inframammary sulcus (79.8\%) in the CC and MLO incidences, respectively, were the most frequent. Considering the positioning criteria evaluated in the MLO incidence, the examinations performed in the private network presented a higher risk of failures related to the nipple centered (RR 4.66, 95\%CI 1.05-20.62, p=0.02) and the visualization of the retro-mammary fat (RR 4.14; 95\% CI 0.92-18.66, $\mathrm{p}=0.04)$, in relation to the exams performed in the public network. Conclusion: The mammograms analysed presented an inadequate quality pattern, with predominance of non-compliance related to breast positioning. 